\title{
Rapid microwave-enhanced, solventless desilylation on potassium fluoride doped alumina
}

\author{
George W. Kabalka*, Lei Wang, and Richard M. Pagni \\ Departments of Chemistry and Radiology, The University of Tennessee, \\ Knoxville, TN 37996-1600 USA \\ E-mail:kabalka@utk.edu
}

Dedicated to Prof. Alfred Hassner on the occasion of his $70^{\text {th }}$ birthday

(received 09 Mar 01; accepted 20 Sep 01; published on the web 28 Sep 01)

\begin{abstract}
A microwave-enhanced desilylation on potassium fluoride doped alumina in the absence of solvents has been developed. The reaction produces the corresponding hydrocarbon products in good to excellent yields.
\end{abstract}

Keywords: Desilylation, fluoride, alumina, microwave

\section{Introduction}

Terminal alkynes are very important in synthetic organic chemistry. They are widely used as reactants in Sonogashira, ${ }^{1}$ Glaser, ${ }^{2}$ hydroboration, ${ }^{3}$ haloboration, ${ }^{4}$ diboration ${ }^{5}$, and various coupling reactions. ${ }^{6}$ The general route to terminal alkynes involves a Sonogashira-Hagihara coupling of (trimethylsilyl)ethyne with aryl halides in the presence of base with a $\mathrm{Cu}(\mathrm{I}) / \mathrm{Pd}(0)$ cocatalyst followed by de-protection of the silyl group carried out using bases, such as potassium carbonate, potassium hydroxide or potassium fluoride in methanol solution. ${ }^{7}$ The reagents and solvents often pose waste handing problems.

We have found alumina to be a particularly useful reagent in organic synthesis because it can be modified in a variety of ways that enhance its reactivity. It also obviates a number of environmental problems. ${ }^{8}$ For example, using a commercially available alumina potassium fluoride mixture to which we added palladium powder, we were able to carry out Suzuki and Sonogashira coupling reactions on a wide variety of aromatic moieties without the use of solvent. ${ }^{9}$ 
Microwave irradiation of organic reaction has gained in popularity in recent years since it was found to accelerate a wide variety of transformations. ${ }^{10}$ Early experiment utilized solvents with high dielectric constants, which permitted rapid heating of reaction solutions. In recent years, a number of reports have appeared in which reactants are coated onto surfaces which themselves absorb little or no microwave energy; in these instances, the reactive species absorb the microwave energy but the temperature of the reaction mixture tends to rise only modestly. This results in relatively large energy savings as well as making it possible to carry out the reaction in simple glassware, such as open beakers and flasks. ${ }^{11}$

We now wish to report a microwave-enhanced desilylation reaction on potassium fluoride doped alumina in the absence of solvents which produces the corresponding hydrocarbon products in excellent yields.

\section{Results and Discussion}

The desilylation reaction is extremely facile for silylated-alkynes, Figure 1. The results are summarized in the Table. The data indicate that, under microwave irradiation in solvent free conditions, desilylation of 2-subsitituted-1-(trimethylsilyl)ethynes readily occurs to yield the corresponding terminal alkynes in excellent yields in the presence of potassium fluoride doped alumina. Substituents on the benzene ring are unaffected by the cleavage reaction. The reaction does not occur in the absence of potassium fluoride (entry a). Microwave irradiation accelerates the reaction and shortens the reaction time from several hours to 1 minute (entry a). The reaction can be utilized to desilylate aromatic and heterocyclic (entries $\mathrm{k}$ and $\mathrm{m}$ ) but not aliphatic derivatives (entry 1$)$. 


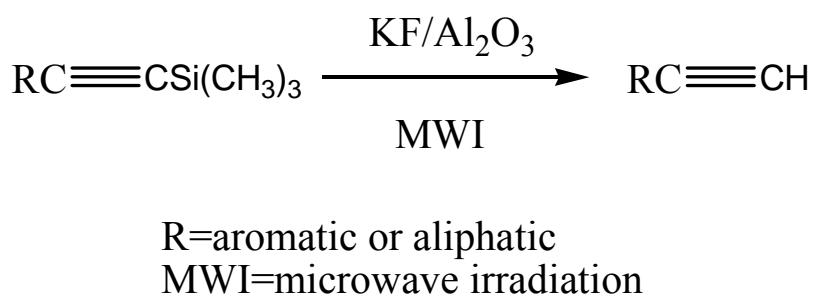

Figure 1. Desilylation of trimethylsilylethynes.

Table . Desilylation of silanes on potassium fluoride doped alumina ${ }^{a}$

\begin{tabular}{lll}
\hline Entry & $\mathrm{R}-\mathrm{Si}(\mathrm{CH} 3) 3$ & Yield $(\%) \mathrm{b}$ \\
\hline & & 90 \\
$\mathrm{a}$ & $\mathrm{Si}(\mathrm{CH} 3) 3$ & $88 \mathrm{c} 0 \mathrm{~d}$ \\
$\mathrm{~b}$ & $\mathrm{Si}(\mathrm{CH} 3) 3$ & 92 \\
\hline
\end{tabular}

${ }^{a}$ Reactions microwaved for 1 minute. b. Isolated yield. c. Yield is based on the reaction at $50{ }^{\circ} \mathrm{C}$ for $4 \mathrm{hrs}$. d. In the absence of potassium fluoride e. Microwave irradiation at $100 \%$ power for $3.5 \mathrm{~min}$.

\section{Experimental Section}

General Procedures. Melting points were recorded on a MEL-TEMP melting point apparatus and are uncorrected. All ${ }^{~} \mathrm{H}-$ and ${ }^{13} \mathrm{C}-\mathrm{NMR}$ spectra were recorded on a $250 \mathrm{MHz}$ Bruker AC 250 spectrometer at $25^{\circ} \mathrm{C}$ using $\mathrm{CDCl}_{3}$ as solvent. Chemical shift are given $\delta$ value with reference to tetramethylsilane (TMS) as internal standard. Coupling constants are given in $\mathrm{Hz}$ and without sign. GC/MS data were obtained by using a Hewlett-Packard 6890 series GC equipped with a 5973 mass selective detector. HRMS were obtained using a VG analytical ZAB-SEQ4F mass spectrometer. A commercially available Sharp Model R-4A38, 1000 watts microwave oven was used. $\mathrm{KF} / \mathrm{Al}_{2} \mathrm{O}_{3}$ ( $40 \%$ by weight) was purchased from Aldrich Chemical Co. Products were purified by flash chromatography on $230-400$ mesh ASTM $60 \AA$ A silica gel.

\section{General procedure for desilylation. Synthesis of phenylacetylene}

1-Phenyl-2-(trimethylsilyl)acetylene $(0.870 \mathrm{~g}, 5.00 \mathrm{mmol})$ was added to $\mathrm{KF} / \mathrm{Al}_{2} \mathrm{O}_{3}(3.00 \mathrm{~g}, 40 \%$ by weight) contained in a $25 \mathrm{~mL}$ round-bottomed flask. The mixture was stirred at room temperature to ensure efficient mixing. The flask was then fitted with a septum which had been punctured by an 18 gauge needle (to serve as a pressure relief valve), placed in the microwave oven and irradiated at $100 \%$ power for 1 minute. After cooling, hexane $(10 \mathrm{~mL})$ was added and 
the slurry stirred at room temperature to ensure product removal from the surface. The mixture was vacuum filtered and the product purified by flash chromatography (hexane as eluent) to yield phenylacetylene.

Phenylacetylene. Oil; ${ }^{12}{ }^{1} \mathrm{H}-\mathrm{NMR} \delta$ 7.49-7.45 (2H, m), 7.27-7.24 (3H, m), $3.04(1 \mathrm{H}, \mathrm{s}) ;{ }^{13} \mathrm{C}-$ NMR $\delta 132.04,128.68,128.22,122.09,83.60,77.24 ; \mathrm{MS} \mathrm{m} / \mathrm{z}$ (relative intensity) $102\left(\mathrm{M}^{+}, 100\right)$, 76 (26), 63 (6), 50 (12).

p-Methylphenylacetylene. Oil; ${ }^{13}{ }^{1} \mathrm{H}-\mathrm{NMR} \delta 7.36(2 \mathrm{H}, \mathrm{d}, J=8.00), 7.07(2 \mathrm{H}, \mathrm{d}, J=7.88), 3.00$ $(1 \mathrm{H}, \mathrm{s}), 2.30(3 \mathrm{H}, \mathrm{s}) ;{ }^{13} \mathrm{C}-\mathrm{NMR} \delta 138.80,131.94,128.98,119.03,83.78,76.48,21.35 ; \mathrm{MS} \mathrm{m} / \mathrm{z}$ (relative intensity) $116\left(\mathrm{M}^{+}, 78\right), 115$ (100), $89(11), 63$ (10).

o-Fluorophenylacetylene. Oil ${ }^{; 4}{ }^{1} \mathrm{H}-\mathrm{NMR} \delta$ 7.50-7.43 (1H, m), 7.32-7.26 (1H, m), 7.10-7.02 $(2 \mathrm{H}, \mathrm{m}), 3.29(1 \mathrm{H}, \mathrm{s}) ;{ }^{13} \mathrm{C}-\mathrm{NMR} \delta 163.25(\mathrm{~d}, J=250.6), 133.97,130.52(\mathrm{~d}, J=7.6), 123.88$, $115.48(\mathrm{~d}, J=20.3), 110.65(\mathrm{~d}, J=15.8), 82.38,76.98 ; \mathrm{MS} \mathrm{m} / \mathrm{z}$ (relative intensity) 120 (M+, 100), 100 (11), 94 (17), 74 (14).

4-Acetylphenylacetylene. $\mathrm{mp} 68-70{ }^{\circ} \mathrm{C}$ (lit. $\left.{ }^{7 \mathrm{a}} 69-70{ }^{\circ} \mathrm{C}\right) ;{ }^{1} \mathrm{H}-\mathrm{NMR} \delta 7.90(2 \mathrm{H}, \mathrm{d}, J=8.32)$, $7.57(2 \mathrm{H}, \mathrm{d}, J=8.43), 3.27(1 \mathrm{H}, \mathrm{s}), 2.59(3 \mathrm{H}, \mathrm{s}) ;{ }^{13} \mathrm{C}-\mathrm{NMR} \delta 197.10,136.71,132.20,128.10$, $126.83,82.69,80.33,26.52 ; \mathrm{MS} \mathrm{m} / \mathrm{z}$ (relative intensity) $144\left(\mathrm{M}^{+}, 31\right), 129$ (100), 101 (54), 75 (21).

o-Ethynylphenol. Oil; ${ }^{15}{ }^{1} \mathrm{H}-\mathrm{NMR} \delta 7.38(1 \mathrm{H}, \mathrm{d}, J=7.51), 7.27(1 \mathrm{H}, \mathrm{dt}, J=7.65, J=1.04), 6.95$ $(1 \mathrm{H}, \mathrm{d}, J=8.20), 6.87(1 \mathrm{H}, \mathrm{t}, J=7.48), 5.80(1 \mathrm{H}, \mathrm{s}, \mathrm{br}), 3.46(1 \mathrm{H}, \mathrm{s}) ;{ }^{13} \mathrm{CNMR} \delta 157.36,132.04$, $130.94,120.34,114.82,108.25,84.34,78.27 ; \mathrm{MS} \mathrm{m} / \mathrm{z}$ (relative intensity) $118\left(\mathrm{M}^{+}, 100\right), 89$ (44), $63(21)$.

o-Ethynylaniline. Oil; ${ }^{16}{ }^{1} \mathrm{H}-\mathrm{NMR} \delta 7.30(1 \mathrm{H}, \mathrm{dd}, J=7.51, J=1.47), 7.08(1 \mathrm{H}, \mathrm{dt}, J=7.74, J=$ 1.41), 6.65-6.58 (2H, m), 4.19 (1H, s, br), 3.35 (1H, s); ${ }^{13} \mathrm{C}$ NMR $\delta 148.38,132.34,129.92$, 117.48, 114.12, 106.25, 82.46, 80.52; MS m/z (relative intensity) $117\left(\mathrm{M}^{+}, 100\right), 90$ (55), 89 (50), 63(17).

p-Acetyl-o-ethynylphenol. mp $100-102{ }^{\circ} \mathrm{C} ;{ }^{1} \mathrm{H}-\mathrm{NMR} \delta 8.05(1 \mathrm{H}, \mathrm{d}, J=1.53), 7.91(1 \mathrm{H}, \mathrm{d}, J=$ 8.72), $7.37(1 \mathrm{H}, \mathrm{s}), 7.03(1 \mathrm{H}, \mathrm{d}, J=8.64), 3.50(1 \mathrm{H}, \mathrm{s}), 2.57(3 \mathrm{H}, \mathrm{s}) ;{ }^{13} \mathrm{C}-\mathrm{NMR}$ 8196.70, 161.57, 133.77, 131.26, 129.64, 115.18, 108.84, 84.39, 77.51, 26.14; MS m/z (relative intensity) 160 $\left(\mathrm{M}^{+}, 40\right), 145$ (100), 117 (48), 89 (31). HRMS Calcd for $\mathrm{C}_{10} \mathrm{H}_{8} \mathrm{O}_{2}: 160.052$. Found: 160.052.

o-Ethynyl-p-methylphenol. Oil; ${ }^{7 \mathrm{c}}{ }^{1} \mathrm{H}-\mathrm{NMR} \delta 7.14(1 \mathrm{H}, \mathrm{s}), 7.01(1 \mathrm{H}, \mathrm{d}, J=8.53), 6.83(1 \mathrm{H}, \mathrm{d}, J$ $=8.41), 5.84(1 \mathrm{H}, \mathrm{s}), 3.39(1 \mathrm{H}, \mathrm{s}), 2.20(3 \mathrm{H}, \mathrm{s}) ;{ }^{13} \mathrm{C}-\mathrm{NMR} \delta 155.07,132.07,131.53,129.43$, 114.60, 107.86, 83.78, 78.55, 20.07; MS m/z (relative intensity) $132\left(\mathrm{M}^{+}, 100\right), 131$ (56), 104 (21), 103 (34), 78 (27), 77 (25).

1-Decyne. Oil ${ }^{17}{ }^{1} \mathrm{H}-\mathrm{NMR} \delta 2.19-2.14(2 \mathrm{H}, \mathrm{m}), 1.91(1 \mathrm{H}, \mathrm{t}, J=2.02), 1.55-1.28(12 \mathrm{H}, \mathrm{m}), 0.88$ $(3 \mathrm{H}, \mathrm{t}, J=6.84) ;{ }^{13} \mathrm{C}-\mathrm{NMR} \delta 84.57,67.99,31.83,29.17,29.09,28.77,28.51$,

22.63, 18.37, 14.00; MS m/z (relative intensity) $137\left(\mathrm{M}^{+}-1,1\right), 109$ (6), 95 (25), 81 (100), 67 (87), 55 (72).

1-Octyne. Oil; ${ }^{18}{ }^{1} \mathrm{H}-\mathrm{NMR} \delta 2.20-2.14(2 \mathrm{H}, \mathrm{m}), 1.92(1 \mathrm{H}, \mathrm{t}, J=2.32), 1.58-1.30(8 \mathrm{H}, \mathrm{m}), 0.90$ 
$(3 \mathrm{H}, \mathrm{t}, J=6.43) ;{ }^{13} \mathrm{C}-\mathrm{NMR} \delta 84.61,67.97,31.29,28.42$ (2C), 22.49, 18.35, 13.92; MS m/z (relative intensity) $109\left(\mathrm{M}^{+}-1,3\right), 95$ (21), 81 (100), 67 (77), 55 (61).

Bromobenzene. Oil; ${ }^{19}{ }^{1} \mathrm{H}-\mathrm{NMR} \delta 7.48-7.39(2 \mathrm{H}, \mathrm{m}), 7.22-7.12(3 \mathrm{H}, \mathrm{m}) ;{ }^{13} \mathrm{C}-\mathrm{NMR} \delta 131.39$, 129.89, 126.72, 122.42; MS m/z (relative intensity) 158, $156\left(\mathrm{M}^{+}, 97,100\right), 77$ (100), 51 (26).

Benzotrizole. mp 98-99 ${ }^{\circ} \mathrm{C}$ (lit. ${ }^{20} 98{ }^{\circ} \mathrm{C}$ ); ${ }^{1} \mathrm{H}-\mathrm{NMR} \delta$ 7.97-7.94 (2H, m), 7.40-7.39 (2H, m); ${ }^{13} \mathrm{C}-\mathrm{NMR} \delta 138.78,126.00,114.85 ; \mathrm{MS} \mathrm{m} / \mathrm{z}$ (relative intensity) $119\left(\mathrm{M}^{+}, 100\right), 91$ (83), 64 (78), 52 (27).

\section{Acknowledgements}

We wish to thank the US Department of Energy and the Robert H. Cole Foundation for support of this research. 


\section{References and Notes}

1. (a) Sonogashira, K.; Tohda, Y.; Hagihara, N. Tetrahedron Lett. 1975, 4467. (b) Nicolaou, K. C.; Dai, W.-M. Angew. Chem., Int. Ed. 1991, 30, 1387. (c) Jones, G. B.; Wright, J. M.; Plourde, II. G. W.; Hynd, G.; Huber, R. S.; Mathews, J. E. J. Am. Chem. Soc. 2000, 122, 1937. (d) Sonogashira, K. In Comprehensive Organic Synthesis; Trost, B. M.; Fleming, I. Eds.; Pergamon Press: New York, 1991; Vol. 3, p 521.

2. (a) Amatore, C.; Blart, E.; Genet, J. P.; Jutand, A.; Lemaire-Audoire, S.; Savignac, M.

J. Org. Chem. 1995, 60, 6829. (b) Li, J.; Jiang, H. J. Chem. Soc., Chem. Commun. 1999, 2369.

3. Brown, H. C. Organic Syntheses via Boranes, Wiley: New York, 1975.

4. Suzuki, A. Pure Appl. Chem. 1986, 58, 629.

5. (a) Ishiyama, T.; Matsuda, N.; Miyaura, A.; Suzuki, A. J. Am. Chem. Soc. 1993, 115, 11080.

(b) Ishiyama, T.; Matsuda, N.; Murata, M.; Ozawa, F.; Miyaura, N.; Suzuki, A. Organometallics 1996, 15, 713.

6. Bottcher, A.; Becker, H.; Brunner, M.; Preiss, T.; Henkelmann, J.; Bakker, C. D.; Gleiter, R. J. Chem. Soc., Perkin Trans. 1 1999, 3555.

7. (a) Takahashi, S.; Kuroyama, Y.; Sonogashira, K.; Hagihara, N. Synthesis 1980, 627.

(b) Austin, W. B.; Bilow, N.; Kellegan, W. J.; Lau, K. S. Y. J. Org. Chem. 1981, 46, 2280.

(c) Arcadi, A.; Cacchi, S.; Rasario, M. D.; Fabrizi, G.; Marinelli, F. J. Org. Chem. 1996, 61, 9280.

8. (a) Kabalka, G. W.; Pagni, R. M. Tetrahedron 1997, 53, 7999. (b) McGinnis, M. B.; Vagle, K.; Green, J. F.; Tan, L.-C.; Palmer, R.; Siler, J.; Pagni, R. M.; Kabalka, G. W.

J. Org. Chem. 1996, 61, 3496. (c) Sponholtz, W. R. III.; Pagni, R. M.; Kabalka, G. W.; Green, J. F.; Tan, L.-C. J. Org. Chem. 1991, 56, 5700. (d) Pala Wilgus, C.; Downing, S.; Molitor, E.; Bains, S.; Pagni, R. M.; Kabalka, G. W. Tetrahedron Lett. 1995, 36, 3469.

9. (a) Kabalka, G. W.; Pagni, R. M.; Hair, C. M. Org. Lett. 1999, 1, 1423. (b) Kabalka,

G. W.; Pagni, R. M.; Wang, L.; Namboodiri, V.; Hair, C. M. Green Chem. 2000, 2, 120. (c) Kabalka, G. W.; Wang, L.; Namboodiri, V.; Pagni, R. M. Tetrahedron Lett. 2000, 41, 5151.

10. (a) Abramovich, R. A. Org. Prep. Proc. Int. 1991, 23, 683. (b) Blettner, C. G.; Koenig, W. A.; Wilfried, A.; Stenzel, W.; Schotten, T. J. Org. Chem. 1999, 64, 3885.

(c) Bose, D. S.; Jayalakshmi, B. J. Org. Chem. 1999, 64, 1713.

11. Varma, R. S. Green Chem. 1999, 1, 43 and references cited therein.

12. Fujii, A.; Miller, S. I. J. Am. Chem. Soc. 1971, 93, 3694.

13. Lambert, J. B.; Larson, E. G.; Bosch, R. J.; Vrucht, M. L. E. T. J. Amer. Chem. Soc. 1985, 107, 5443.

14. Mal'kina, A. G.; Brandsma, L.; Vasilevsky, S. F.; Trofimov, B. A. Synthesis 1996, 589.

15. Alberola, A.; Calvo, B.; Ortega, A. G.; Pedrosa, R. J. Chem. Soc., Perkin Trans. 1 1992, 
3075.

16. Topolski, M. J. Org. Chem. 1995, 60, 5588.

17. Taherirastgar, F.; Brandsma, L. Synth. Commun. 1997, 27, 4035.

18. Nelson, D. J.; Blue, C. D.; Brown, H. C. J. Am. Chem. Soc. 1982, 104, 4913.

19. Kotsuki, H.; Datta, P. K.; Hayakawa, H.; Suenaga, H. Synthesis 1995, 1348.

20. Velino, B.; Cane, E.; Gagliardi, L.; Trombetti, A.; Caminati, W. J. Mol. Spectrosc. 1993, $161,136$. 